\title{
Socially Respectful Enjoyment Tracking for Tabletop Games
}

\section{Ben Kirman}

University of Lincoln

Brayford Pool, Lincoln UK

bkirman@lincoln.ac.uk

\section{Duncan Rowland}

University of Lincoln

Brayford Pool, Lincoln UK

drowland@lincoln.ac.uk

\begin{abstract}
This paper describes the current state of our ongoing work developing tools for tracking player enjoyment in traditional face-to-face tabletop gaming situations. The challenge presented is that of quantifying game enjoyment whilst minimising the effects of the measurement techniques on the validity of the study. This paper presents the development of a self-report tool for cellular phones that aims to gauge player enjoyment with minimal impact on the "magic circle" of play. Its design for non-invasive and meaningful selfreport data is described along with initial preliminary results from casual trials that indicate the potential value of the technique along with avenues for further study.
\end{abstract}

\section{Keywords}

Ecological Validity, Magic Circle, Flow, Fun,

Entertainment, Board Games

\section{ACM Classification Keywords}

K.8.0 [Computing Milieux]: Personal Computing Games, ].4 [Social and Behavioural Sciences]:

Psychology

\section{Introduction}

Copyright is held by the author/owner(s).

CHI 2008, April 5 - April 10, 2008, Florence, Italy ACM 978-1-60558-012-8/08/04.
The appreciation of a user's transient mental mood state could be important to the design of interfaces in the future. Tabletop gaming provides an ideal vehicle 
with which to investigate the acquisition such subjective data. This paper describes our initial progress in using cell phones to facilitate the self-report of this information in a socially unobtrusive yet reliable manner.

The face-to-face social gaming environment (exhibited by traditional tabletop gaming) has major differences to that of video games. The most profound is the absence of a computer mediating the experience. Instead, the players themselves are directly responsible for the operation and management of the game.

Work by other researchers on video games is concerned with using computer-based solutions for gauging player enjoyment (e.g. FUGA [5]). By contrast, our assumption is that for tabletop games, the measuring device should be as invisible as possible and not introduce technological elements to an environment where there were none previously.

In this case, it is proposed that cellular phones make an ideal platform for sampling tools since they are practically invisible in today society (camouflaged by their ubiquity). While a larger computer based system would be out of the ordinary in a social gaming

situation, cellular phones are so common that they can be present in the gaming environment without causing disruption to it.

\section{Protecting the Magic Circle}

Play is a highly subjective and personal experience and when it is observed the method of study can directly affect the level of enjoyment the player experiences. Since play is by definition outside the normal activities of survival, "essentially a separate occupation, carefully isolated from the rest of life" [1], menial tasks such as filling out questionnaires, or indeed wearing bulky physiological measuring devices, intrude into the experience and may interrupt the supposed immersive "flow" [4] state reached during play.

"The Magic Circle" is used to describe the change in cognitive context players enter when playing games [7] - How the pieces on the board or pixels on the screen take on new and important meanings with specific rules and attributes that aren't applied outside the game. As Salen and Zimmerman [11] note, some magic circles are literal - the stage in a theatre, the railings around a park or the boundary on a sports pitch; others are conceptual, requiring the suspension of disbelief to occur within the boundaries of the set of agreed rules.

\section{Less Intrusive Sampling Tools}

In order to reduce the impact on the magic circle the platform needs to integrate with the typical face-to-face social gaming ecology as seamlessly as possible. Given the power of self-report as a tool for measuring spontaneous activities [6],[9], it would appear an appropriate method to apply in the study of player enjoyment during game play.

Due to the ubiquity and computing power available in modern cellular phones, they make the ideal platform for building an ecologically respectful sampling device. They are so pervasive in modern society that it is usual to find them already within existing gaming environments. Users are thus likely to be comfortable with manipulating their phone in a play environment and, of utmost importance; it is seen as socially acceptable to do so. Testing the validity of this 
assumption through the research will show the actual value of cell phones as in-play research tools.

\section{Scale Selection}

Questionnaires are only suitable for studying play while the subject is not in the magic circle due to the high degree of focus required and the introspective nature of this form of data collection. They are likely best used in addition to continuous data collection throughout the game activity. In-play self-report tools must be fast to interact with and they must be simple to use. The longer a player spends introspecting and recording their level of enjoyment, the further they drift from their mental play state.

For in-play self-reporting (In addition to longer post study questionnaires), the simplest interface poses a single question that the participant answers many times either at prompted moments during the experiment or whenever they feel a change in state has occurred. A straightforward example is the question "How much are you enjoying playing this game at this moment?" with an answer provided using a Likert scale.

\section{Prompting for Reports}

Since the aim of the self-report tool is to disrupt the flow of the game as little as possible, it seems likely that the players will simply forget to report due to being immersed into the game (i.e. in a flow state [4]).

Prompting the user every few minutes to remind them about the tool can interrupt the flow of the game as defined by its rules [8], changing the nature of the experience if they occur during moments of concentration. Initial observations indicate that this kind of random prompt may be unnecessary: the players may self-prompt based on their status within the game and even based on social factors.

\section{Preliminary Studies}

As part of a research study into the dynamics of the play experience in face-to-face board games, several prototype self-report tools for mobile devices were created. These were tested casually with a local group of board game enthusiasts during regular play sessions.

Each prototype was developed in J2ME for deployment on cell phones and implemented a different scale and input style. All prototypes allowed the users to choose the moment when to record a state self-report, which would be logged against a timestamp for analysis.

\section{Prototype I}

The first prototype tested a form of input to track relative change in enjoyment. Players were presented with 5 options from which to choose the rate of change.

Players were asked to track their enjoyment in relative terms, so were expected to log the relative change in enjoyment as it happened through the game.

Users reported feeling confused by the relative reporting. They generally had trouble keeping in mind their level of enjoyment between reports - in these cases they were unable to remember their previous state in order to be able to

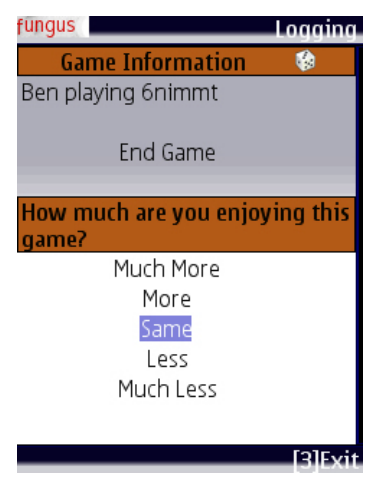

Figure 1 - Prototype I 
decide the appropriate relative change.

\section{Prototype II}

The second prototype used absolute input to track enjoyment state. Players were asked to select a value that represented their level of enjoyment on a 7 point Likert scale, where 1 was the least enjoyment and 7 was the most.



Figure 2 - Prototype II
Prototype III

An extension of Prototype II, this prototype used the same system except with a 9 point Likert scale, 9 being the most enjoyment. As an enhancement, players were able to enter the report using the numerical keypad on the phone rather than navigating a menu on the screen. The users reported that the keypad was the preferred choice in this case. In addition, due to the screen-saver feature of the phones, when using the joystick users found that they could submit a report in error when they were simply trying to "wake up" the device after the screen saver had become active.

\section{Prototype IV}

The final prototype used a slider on a 100-point scale to allow the players great freedom when reporting level of enjoyment using the cell phone joystick. Participants showed a strong preference for the button input. Moving a slider up and down was only good for recording small changes in state. Pressing a button allowed them to record any state at great speed. Also, there were concerns

about privacy of reports.

When a user moves the

slider, a feature of the phone keeps the screen lit up for several seconds after the interaction. In this time, it is possible for other players to briefly see the level of the slide on the phone screen and therefore see the current enjoyment state of the reporting player.



Figure 3 - Prototype IV

\section{Initial Observations}

The objective of the casual trials was to identify problems with the usability of the tools and gain insights into the effectiveness of each method. Based on observations and comments from the trial participants, a reporting tool will be created that will be used for formal experimentation under more controlled circumstances.

\section{Reporting Patterns}

As a side effect of the casual trials, the data indicated some interesting behaviours regarding the need to prompt. A common comment from the volunteers was that they thought that the tool needed some form of prompting to remind them to report their state and on at least two occasions testers apologised for not having used the tool very much. These comments, however, did not match up with the reality of the data recorded in the session. In 19.9 player hours of gaming that occurred during the tests, the tools recorded an average of 10.95 self-reports per player per hour. 
This contradiction between what self-report subjects recall and the reality has been previously documented with smokers [12] and alcoholics [2], and will be explored with gamers as part of the formalised research of which these tests formed the groundwork.

\section{GAME FLow PROMPTING}

Observations during the initial trials showed that, in games featuring player turns, players would rarely submit a report during their turn, and it was common for a player to submit a report soon after their turn was over. This indicates the players make conscious decisions to report at points during the game where they are least involved.

AUTO-SOCIAL SYNCHRONISATION OF REPORTS The data from the casual trials indicate that despite players being able to report at any time they wish, typically player reports happened within a few seconds of another player submitting a report.

This is a natural result of the type of games being played. The tests were with card and board games that were played on a central shared surface. When one player picks up the cell phone in order to make a report, the movement associated with this activity caught the attention of the other players, who would be reminded to also pick up their device and report.

This behaviour can be seen clearly on the graph in figure 4 . The group is playing a trivia game [3] that consists of seven rounds of questions to be answered by players simultaneously. Once all players have written an answer, they are revealed and players can place bets on which answer they think is nearest before the correct answer is revealed.

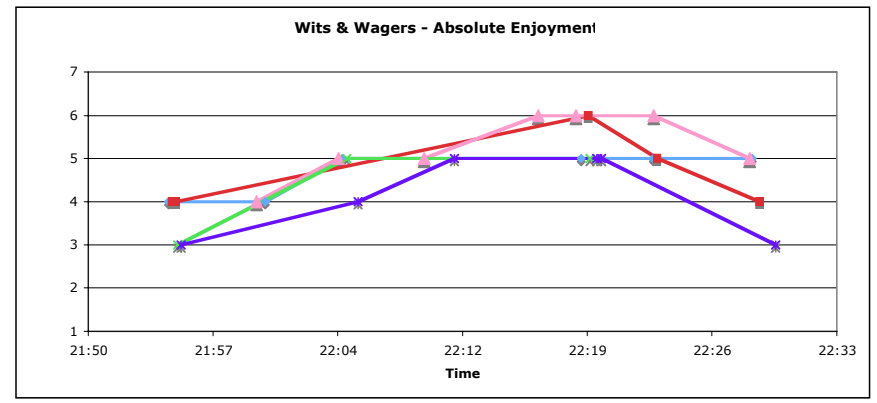

Figure 4 - Trial Results for Wits \& Wagers using Prototype II

Given the players could choose to report mood at any point during the game, there appears to be seven clusters of reports during the game. This suggests a combination of game flow and social synchronisation led to the timing of these reports, during a game where any intentional prompts were absent.

\section{Discussion \& Future Work}

These studies are designed to focus on ecological validity as a key factor to take into consideration when designing tools for measuring highly subjective variables such as enjoyment in games. Whatever method is chosen will inevitably affect the game experience for the subjects of the experiment through invasiveness or simple novelty. The design of the studies presented are particularly aware of the fragile psychological play/flow state that gamers enter when playing games, and are careful not to break the magic circle of play.

A simple self-report tool for measuring enjoyment using cellular phones has been presented. Cell phones are truly ubiquitous devices in modern society, and so pervasive that it is not unusual to find the devices 
present during normal play sessions. Due to this familiarity it is proposed that the effect on the magic circle will be minimal.

As a result of the short series of casual trials carried out with the self-report prototypes, several potential areas for further research were uncovered, and will be investigated further.

- How game state and social factors affect frequency and pattern of reports.

- Investigating discrepancies between recalled and actual report frequencies.

- How the game experience is changed by the act of study.

Further studies are planned in which participants are used to gather second order information - this is, rating the perceived state of other players. This will enable the comparison of actual reported and perceived values to be correlated of for a determination of the accuracy of the technique to be assessed.

\section{Acknowledgements}

This study is funded under the social gaming strand of the EU FP6 PASION project

Thanks to the Lincoln Board Games Group [10] for testing the prototypes and providing valuable feedback.

\section{References}

[1] Caillois R., Man, Play and Games, The Free Press of Glencoe, 1961
[2] Collins R.L., Kashdan T.B. and Gollnisch G., The Feasibility of Using Cellular Phones to Collect EMA Data: Application to Alcohol Consumption, In Experimental and Clinical Psychopharmacology 11.1 pages $73-78,2003$

[3] Crapuchettes, D, 2005, Wits \& Wagers, published by North Star Games,

http://www.northstargames.com/games/wits_and_ wagers/

[4] Csikszentmihaly M., Flow: The Psychology of Optimal Experience, Harper Collins, 1990

[5] Fun of Games: The FUGA Project, http://project.hkkk.fi/fuga/

[6] Gee P., Coventry K.R. and Birkenhead D., Mood state and gambling: Using mobile telephones to track emotions, In the British Journal of Psychology 96 pages 53-66, 2005

[7] Huizinga J., Homo Ludens: A Study of the Play Element in Culture, Beacon Press, 1955

[8] Juul, J., Half-Real: Video Games between Real Rules and Fictional Worlds, MIT Press, 2005

[9] Lee G., Raab F. et al, PmEB: A Mobile Phone Application for Monitoring Caloric Balance, Proceedings of $\mathrm{CHI} 2006$

[10] Lincoln Board Games Group, http://www.lincolnboardgames.org.uk

[11] Salen K. and Zimmerman E., Rules of Play: Game Design Fundamentals, MIT Press, 2003

[12] Schiffman S., Hufford M. et al, Remember that? A comparison of real-time versus retrospective recall of smoking lapses, Journal of Consulting and Clinical Psychology, 65, pages 292-300, 1997 